\section{Cometary collisions on the Moon and Mercury}

IT has been proposed that unusual bright and dark swirl-like markings on the Moon and Mercury may be strongly magnetized residues of recent $(<100 \mathrm{Myr})$ collisions of the latter bodies with one or more cometary comas ${ }^{1}$. The photogeological investigations which led to this hypothesis were stimulated, at least in part, by emerging evidence that relatively high-amplitude magnetic anomalies detected with instruments on-board the Apollo subsatellites are concentrated in areas where swirls are found ${ }^{2,3}$. Although we agree that many of the swirls are probably relatively strongly magnetized deposits of impact-generated material, we consider that meteoroid impacts occurring over a span of time comparable to the age of the Solar System can explain their existence at least as well as can recent comet impacts. Our reasons are given below.

First, the inferred sources of mediumamplitude magnetic anomalies on the central near side ${ }^{2,3}$ and on the far side ${ }^{4}$ are basin ejecta materials. This result is particularly well established on the near side, where anomalies are correlated with exposed segments of primary Imbrium basin ejecta (the Fra Mauro Formation) surrounded by poorly magnetized mare basalt. Both returned sample studies and surface magnetic field investigations support the contention that ejecta materials produced during meteoroid impacts are major sources of magnetic anomalies detected from orbit ${ }^{5,6}$. Therefore, a special explanation for the highamplitude anomalies involving "highvelocity imprinting of [cometary] coma fine structure" may be ad hoc.

Second, there is photogeological evidence that the Reiner Gamma swirl on western Oceanus Procellarum is related in origin to a nearby $3.2 \pm 0.2 \times 10^{3}$-Myr-old primary impact crater ${ }^{3}$ and that some of the larger swirls in Mare Ingenii are associated with the Copernican (1-2x $10^{3}$ Myrold) crater O'Day? ${ }^{7}$. Thus, a genetic relationship of all the swirls to the late Copernican crater Goddard A caused by a "prograde, incoming split comet system impacting the Moon" as proposed in ref. 1 is arguable. Note also that although there is evidence that many of the more conspicous far side swirls are very young, it is not known whether the young swirls are magnetized.

Third, if both the swirls and the highamplitude magnetic anomalies are caused by comet impacts, magnetic signatures of more ancient (and more numerous) such impacts should have been detected all over the Moon. Instead, virtually all the high-amplitude magnetic anomalies detected between $\pm 30^{\circ}$ of latitude with the Apollo subsatellite magnetometers and charged particle instruments occur in limited selenographic regions where swirl patterns exist. The restricted occurrence of both swirls and high-amplitude magnetic anomalies within particular regions suggests a geological rather than an entirely exogenic control on their origin.

Fourth, the distribution of the largest concentrations of swirl patterns (and magnetic anomalies) on the moon is not random as expected for unusual additions of cometary matter. Specifically, as first pointed out by El-Baz ${ }^{8}$, the most conspicuous concentrations of swirls (north of Mare Marginis and within Mare Ingenii) are roughly antipodal to the two youngest large impact basins, Orientale and Imbrium, respectively. As evidence that the antipodal relationship is not fortuitous, the two additional regional concentrations of swirls mapped in Fig. $3 b$ of ref. 1 (centred at $165^{\circ} \mathrm{W}, 23^{\circ} \mathrm{S}$ and $125^{\circ} \mathrm{W}$, $20^{\circ} \mathrm{S}$ ) are within $5^{\circ}$ of the Serenitatis and Crisium antipodes, respectively. Each of these four areas is known to be anomalously magnetic ${ }^{9,10}$ and at least three of the four areas is marked by the general occurrence of unusual hilly and lineated terrain ${ }^{11,12}$. The latter has been ascribed either to compression at the antipode by body and surface seismic waves generated by the associated basin impacts $^{13}$ or to convergence of basin secondaries at the antipode ${ }^{14}$. To explain the concentrations of both swirls and large magnetic anomalies near basin antipodes, a compositional modification of the crust in areas where the hilly and lineated terrain is found has been postulated ${ }^{15}$ such that subsequent (meteoroid) impacts on these areas result in crater ejecta which is especially susceptible to magnetization. The necessary compositional modification was proposed to be an increase in metallic iron content, as metallic iron is the ferromagnetic carrier in all sampled lunar materials.

\section{L. HOOD}

Lunar and Planetary Laboratory,

University of Arizona,

Tucson, Arizona 85721

1. Schultz, P. H. \& Srnka, L. J. Nature 284, 22 26 (1980). 2. Hood, L. L., Coleman, P. J. Jr \& Wilhelms, D. E. Science 204, 53-57 (1979)

3. Hood, L. L., Coleman, P. J. Jr \& Wilhelms, D. E. Proc. 10th lunar planet. Sci. Conf. 2235-2257 (1979).

4. Anderson, K. A. \& Wilhelms, D. E. Earth planet. Sci. Lett. 46, 107-112 (1979).

5. Strangway, D. W., Gose, W., Pearce, G. \& McConnell, R. K. Nature 246, 112-114 (1973).

6. Fuller, M., Cisowski, S. M. \& Hale, C. J. Proc. 10 th lunar planet. Sci. Conf., 2211-2233 (1979)
7. Schultz, P. H. Moon Morphology, 420 (University of Texas Press, Austin, 1976).

8. El-Baz, F. Trans. Am. Geophys. Un. (EOS) 52, 856 (1971).

9. Lin, R. P., El-Baz, F., Hood, L. L., Runcorn, S. K. \& Schultz, P. H. in Lunar Planet. Sci XI, 626-627 (Lunar and Planetary Institute, Houston, 1980).

10. Hood, L. L., Russell, C. T. \& Coleman, P. J. Jr (in preparation).

11. Wilhelms, D. E. \& El-Baz, F. U.S. Geol. Surv. Map, I-948 (1977).

12. Stuart-Alexander, D. E. U.S. Geol. Surv. Map, I-1047 (1978).

13. Schultz, P. H. \& Gault, P. E. The Moon 12, 159-177 (1975).

14. Moore, H. J., Hodges, C. A. \& Scott, D. H. Proc. Sth lunar Sci. Conf., 71-100 (1974)

15. Hood, L. L. in Conf. Lunar Highlands Crust, 72-74 (Lunar and Planetary Institute, Houston, 1979).

SCHULTZ AND SRNKA REPLY-In response to Hood's comments concerning our proposed origin of the bright and dark swirls on the Moon and Mercury, we make the following comments.

First, Hood's proposal that these swirls are strongly magnetized deposits of ejecta from major cratering events is not consistent with available photogeological data. Second, although it has been argued that strongly magnetized material can be generated by an impact event ${ }^{1-3}$, it is another matter to transport this material in an ordered fashion over long distances so as to create a magnetic anomaly during its deposition; secondary impact processes and local mixing would be expected to randomize the incoming magnetized material and prevent the building of an anomaly. Rather, the ejecta must come to rest and acquire remanence in the presence of a field; this is difficult to accomplish for impact-generated magnetic fields except perhaps for nearrim basin-sized events ${ }^{2}$.

Specific responses to Hood's comments are as follows. First, the existence and possible correlation of medium-amplitude anomalies with the Fra Mauro Formation ${ }^{4}$ (Imbrium ejecta deposits) are irrelevant to discussions of the origin of the swirls, other than to note that there are at least three likely processes responsible for lunar magnetic anomalies: impactinduced magnetization preserved in thick ejecta deposits, remanence acquired in the field of a primordial lunar dynamo, and our proposed cometary magnetization $^{5}$. We stated in our report that all three (and likely more) processes have occurred on the Moon during its evolution, but that only cometary magnetization is consistent with the nature of the swirls.

Second, photogeological data do not show that Reiner Gamma is associated with the crater Cavalerius, contrary to the proposal by Hood et al. ${ }^{4}$. The swirls are surficial in character and cross a variety of features including secondary craters from Cavalerius. Cavalerius is sufficiently old for craters as large as $10 \mathrm{~km}$ to have superposed its ejecta deposits, excavating 